\title{
Assessing the Performance of Variable Stiffness Continuum Structures of Large Diameter
}

\author{
Angus B. Clark Student Member, IEEE and Nicolas Rojas Member, IEEE
}

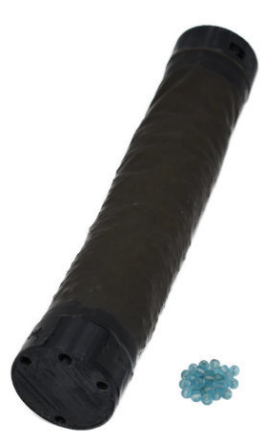

A

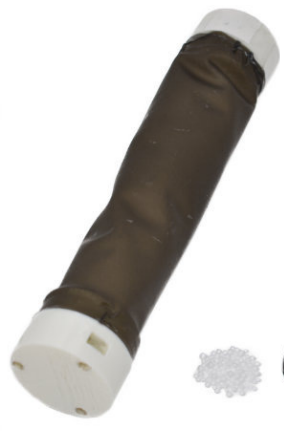

B

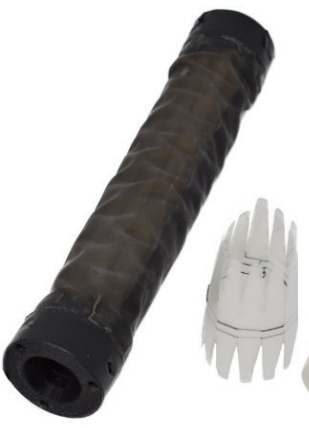

C

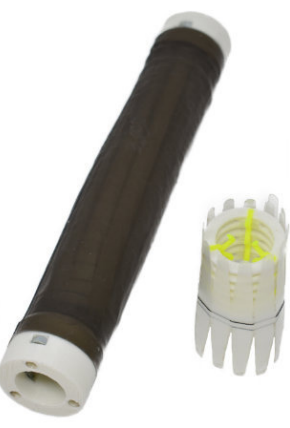

D

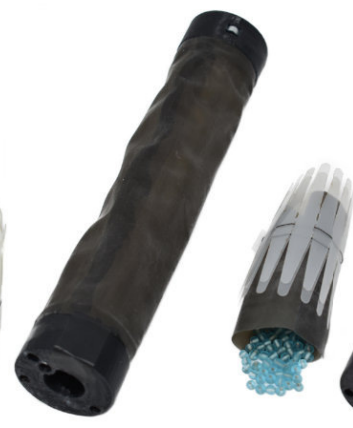

$\mathrm{E}$

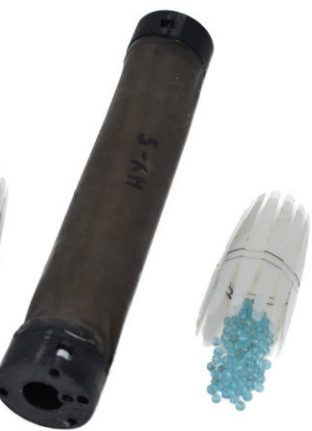

F

$\longmapsto 50 \mathrm{~mm}$

Fig. 1. Large diameter variable stiffness continuum structures based on varying jamming technologies that were compared using the proposed testing methodology. Namely: (A) rigid granules; (B) deformable granules; (C) layer jamming; (D) layer jamming with internal support structure; (E) rigid granules and layer jamming (hybrid) in separate jamming volumes; and (F) rigid granules and layer jamming (hybrid) in a single jamming volume.

Abstract-Variable stiffness continuum structures of large diameter are suitable for high-capability robots, such as in industrial practices where high loads and human-robot interaction are expected. Existing variable stiffness technologies have focused on application as medical manipulators, and as such have been limited to small diameter designs $(\sim 15 \mathrm{~mm})$. Various performance metrics have been presented for continuum structures thus far, focusing on force resistance, but no universal testing methodology for continuum structures that encapsulates their overall performance has been provided. This paper presents five individual qualities that can be experimentally quantified to establish the overall performance capability of a design with respect to its use as a variable stiffness continuum manipulator. Six large diameter $(>40 \mathrm{~mm})$ continuum structures are developed following both conventional (granular and layer jamming) and novel (hybrid designs and structurally supported layer jamming) approaches, and are compared using the presented testing methodology. The development of the continuum structures is discussed, and a detailed insight into the tested quality selection and experimental methodology is presented. Results of experiments demonstrate the suitability of the proposed approach for assessing variable stiffness continuum capability across design.

Index Terms - Soft Robot Materials and Design; Performance Evaluation and Benchmarking; Flexible Robots; Modelling, Control, and Learning for Soft Robots

Manuscript received: October, 12, 2018; Revised January, 29, 2019; Accepted March, 5, 2019.

This paper was recommended for publication by Editor Kyu-Jin Cho upon evaluation of the Associate Editor and Reviewers' comments. This work was supported in part by the Engineering and Physical Sciences Research Council grant EP/R020833/1.

Angus B. Clark and Nicolas Rojas are with the REDS Lab, Dyson School of Design Engineering, Imperial College London, London $\{a . c l a r k 17$, n.rojas\}@imperial.ac.uk

Digital Object Identifier (DOI): see top of this page.

\section{INTRODUCTION}

$\mathbf{S}$ OFT robots have shown the ability to accomplish tasks traditional rigid robots have struggled with, specifically providing safety in human-robot interaction and demonstrating a high level of manipulation without the accompanying control complexity. This has lead to their expansive use in certain areas of robotics including manipulation and grasping [1], [2], medical surgery robotics [3], and exoskeletons [4]. The promising performance of soft continuum robots has led to the development of various continuum implementations [5], [6], [7], however despite providing increased dexterity they suffer from a number of issues, most notably a lack in holding strength [8], [9]. To solve this, soft continuum robots have integrated variable stiffness technologies within their design, allowing for both soft, highly flexible motion and rigid, high load resistance [1], [10], [3]. Herein, we refer to these variable stiffness continuum designs as just continuum designs/structures to facilitate discussion.

Previous (variable stiffness) continuum designs have limited themselves to small $(\sim 15 \mathrm{~mm})$ diameters to allow for use specifically in minimally invasive surgery (MIS) and natural orifice transluminal endoscopic surgery (NOTES), which both impose limitations on the maximum dimensions of the design [11]. Larger diameters have been demonstrated in alternative areas of implementation [12], however these are few and far between when compared to surgery-based designs. Despite following similar testing procedures to establish their performance, designs of varying size are difficult to compare due to performance being strongly related to size and with no clear understanding of scalability of stiffening technologies. When considering the stiffening technologies used, various solutions 


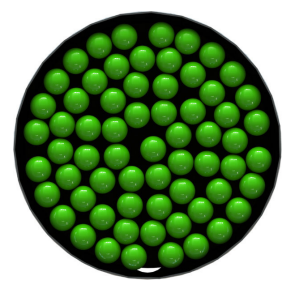

(a)

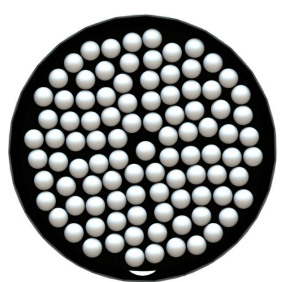

(b)

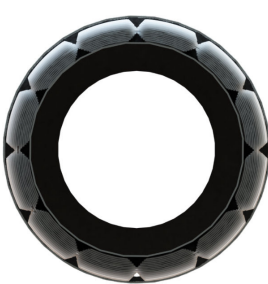

(c)

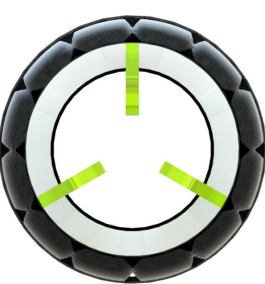

(d)

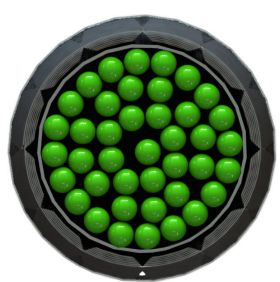

(e)

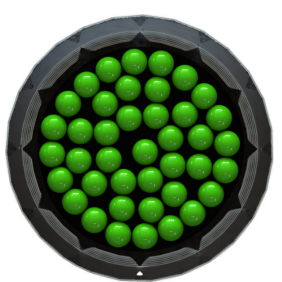

(f)

Fig. 2. Cross sectional view of continuum structures based on granular jamming with rigid granules (a) and deformable granules (b), layer jamming (c), layer jamming with supporting internal structure (d), and hybrid designs containing both rigid granules and layer jamming in individual jamming volumes (e) and a single shared jamming volume (f).

have been presented and are available [11], [13]. Significant progress being shown with mechanically jamming structural interactions due to their simplicity in integration using either tendon wires [14], [15], or vacuum-activated granular or layer jamming, which provide simple control over the stiffness with variation in applied pressure [1], [3].

Tests of continuum structure performance typically focus on the force resistance, defined as the force exerted by the structure as it is deformed perpendicularly a set distance at its furthest end, with base fixed in position. Generally this is performed with the structure rigid with $0^{\circ}$ bending [1], [16], however occasionally it is also performed at $90^{\circ}$ bending [15], [17]. The results of this can then be used to calculate the overall stiffness and stiffening factor of the structure. Other tests analyse the individual properties of the stiffening materials, such as frictional coefficients, however due to variations in stiffening methods these are not comparable across different designs.

Currently, performance is typically defined by the materials and the structure utilised, therefore results require scaling to compare present issues with accuracy and reliability. Further, the limited performance metrics available are currently general in application, and so are poor in optimising a design for a specific area, for example medical robotics or precise manipulation. Ideally, a range of metrics should be available, where the desired properties, and therefore the optimal design, can be selected. These measurements should consider aspects further than solely force resistance, and should include items such as positional accuracy and consider the effect of bending on the structure-elements that are also critical in continuum design.

In this paper we present a methodology for establishing the performance of a continuous design through five metrics, namely: resisting force, bending diameter deformation, physical dimensional properties, positional accuracy, and deformation under external forces. The proposed experimental procedure encapsulates the overall performance of the continuum design, and allows comparison across varying dimensions and stiffening technologies. Further, it allows for optimised selection of design when considering continuum structures for use in robotics in different sectors.

While previously resisting force [2], [16], [1] and bending diameter deformation [18] have been used for performance comparison of continuum structures, and reviews for designs for medical applications considering resisting force and activa- tion time have been produced [11], to the best of the authors' knowledge no comprehensive experimental procedure as this herein proposes has thus far been presented, which provides analysis on the positional accuracy or deformation for instance. To exemplify the presented testing methodology, six large diameter $(>40 \mathrm{~mm})$ continuum structures are developed and compared; these designs follow both conventional (granular and layer jamming) as well as novel (hybrid designs and structurally supported layer jamming) stiffening approaches (see Fig. 1).

The rest of this paper is organised as follows. Section II presents the design and fabrication process of the six variable stiffness continuum structures. In section III we discuss the selection of performance metrics and their experimental procedure, and present the resulting performance of the six developed structures in section IV. Finally, we discuss the experimental results in section $\mathrm{V}$, providing analysis on the continuum structures and reflection on the testing methodology.

\section{FABrication of CONTINUUm Structures}

Six large diameter continuum structures were designed for use as manually positioned manipulators, where a position could be formed by grasping and bending the structure to the desired topology. Typical previous designs employ a small diameter, optimised for use in surgery rather than inhand bending, whereas an optimal grasp diameter would be significantly larger at $\sim 50 \mathrm{~mm}[19]$. As such, we implemented structures with diameters ranging from $41 \mathrm{~mm}$ to $48 \mathrm{~mm}$, to ensure a more optimal experience with manual positioning, which may be needed in industrial settings. By increasing the continuum structure size, limitations were introduced on the stiffening technologies capable of being used. For example, variable stiffness provided using material transitionse.g., shape memory alloys (SMAs), low melting point alloys (LMPAs) — are limited to small structures due to their difficulty to scale, showing large increases in stiffening transition time and a reduced stiffness range as size increases [17], [13]. To allow for the increase in diameter, stiffening technologies were selected based on their simplicity to implement and ability to scale, as this allowed for the focus to be on the testing methodology rather than the continuum structure design. Granular and layer jamming were selected due to their previously demonstrated ability to scale up to larger dimensions [11], 


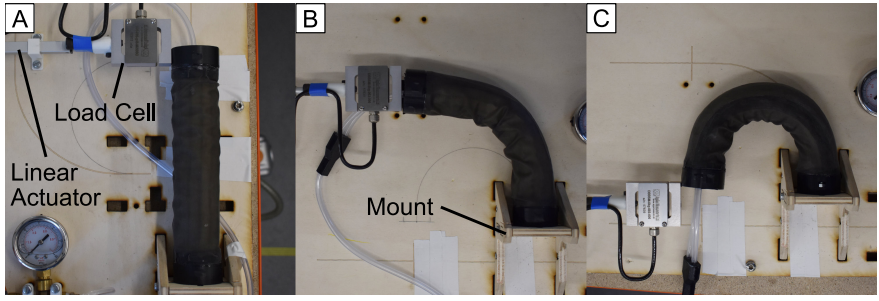

Fig. 3. Experimental setup for determining the resisting force of a continuum structure at (a) $0^{\circ}$, (b) $90^{\circ}$, and (c) $180^{\circ}$ bending positions (layer jamming).

as well as their promising performance with respect to force resistance compared to alternatives.

The continuum structures were designed following a lengthdiameter ratio of $\sim 5$, with a total length of $240 \mathrm{~mm}$. To allow for mounting and fixing the structure in place, as well as to determine a finite length and provide a reference point for testing, rigid ends with mounting points and vacuum tubes were added to each end of the structure, at $20 \mathrm{~mm}$ long each, giving 200mm length of variable stiffness. The stiffening capability was enabled using negative pressure, provided by a vacuum pump (BACOENG $220 \mathrm{~V} / 50 \mathrm{~Hz}$ BA-1 Standard). To allow for stiffening to occur, the stiffening volume was sealed airtight using a membrane, selected as to not affect the bending capability of the continuum structure.

The structures were constructed using $0.25 \mathrm{~mm}$ sheet latex, cut to size and formed into cylinders using liquid latex glue, which was attached to rigid mounts at each end using Ethyl 2cyanoacrylate (Super Glue). The rigid mounts were formed out of acrylonitrile butadiene styrene (ABS) using fused deposition modelling (FDM) 3D printing, which allowed for intricate design including mounting, vacuum tube pass-through, and affixing points for the membrane. To provide the negative pressure, a $6 \mathrm{~mm}$ polyvinyl chloride (PVC) tube connected the structure to a vacuum pump.

The properties for the structures were as follows:

\section{A. Rigid and Deformable Granules}

$4 \mathrm{~mm}$ diameter matt-surfaced glass beads were utilised as the rigid granular filling, based off previous work presenting optimal granule materials and dimensions [1]. The design implemented is shown in Fig. 2(A). Deformable granules show a useful comparison against rigid granules, producing tighter packing (and therefore a higher packing efficiency) when activated as previously presented in [12]. 3mm diameter polystyrene packing beads were used as deformable granules, selected for their stiffness and similar dimensions to the selected rigid granules. The corresponding design implemented is shown in Fig. 2(B). Both granular designs were the easiest to implement, using a single outer membrane to seal the filled volume.

\section{B. Layer Jamming w/o(w/) Supporting Internal Structure}

By wrapping laser cut layers of $0.18 \mathrm{~mm}$ Mylar sheet into a tubular structure, connected by polyester thread, a sheath capable of demonstrating high resisting forces under vacuum due to cumulative frictional forces was produced. Specific details

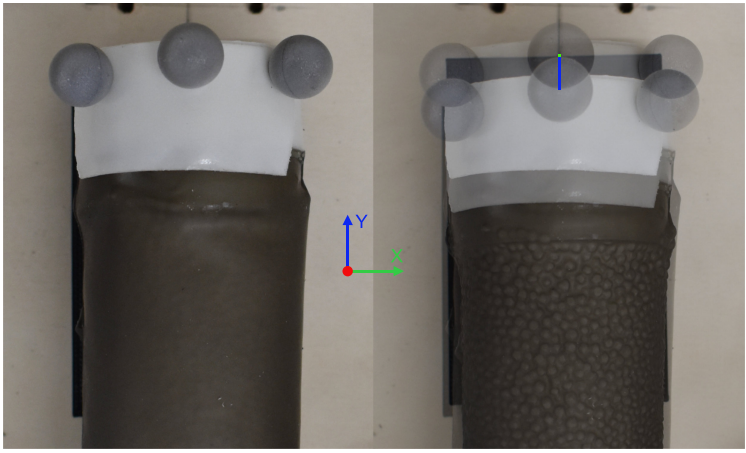

Fig. 4. Positional accuracy variation, showing motion tracked markers and orientation of coordinate plane (deformable granules).

of the layer pattern and dimensions can be found in [18], and alternative designs at smaller diameters following a similar structure can be found in [2], [3], [17]. As layer jamming forms a hollow cylinder, a second internal latex membrane was included, utilised in both layer jamming designs. The second design uses an internal flexible support structure as proposed in [18], which further improves the performance of layer jamming under bending. The implemented layer jamming designs without and with supporting internal structure are shown in Fig. 2(C) and Fig. 2(D), respectively.

\section{Combined Layer Jamming and Rigid Granules}

To test the combined performance of both granular and layer jamming, two novel hybrid designs collaborating both stiffening technologies were developed. Both designs followed a layout of layer jamming forming the outer cylinder, with the inside of the cylinder filled with $4 \mathrm{~mm}$ rigid granules. Differing from both layer jamming structures, only a single outer membrane was used. However, while one hybrid design contained both layer jamming and granular jamming in a single shared volume (hybrid - shared volume, Fig. 2(F)), the other hybrid design contained a second membrane between the two stiffening technologies, separating them into individual stiffening volumes (hybrid - individual volumes, Fig. 2(E)). Both individual volumes were connected to the same vacuum source using a Y-adaptor and PVC tubes.

\section{Evaluation OF CONTINUUM CAPABILITY}

To establish the performance of the developed continuum structures, we present five metrics that can be experimentally quantified. The aim of the assessments is to provide a range of results which help establish the overall performance of a continuum structure, and help to provide comparison without the need for similar size or stiffening technologies. By proposing a general testing methodology, we hope to establish a model for the presented performance of future developed continuum structures. In what follows, each metric along with its corresponding testing methodology is detailed.

\section{A. Test 1: Resisting Force}

Already mentioned as a weak area of continuum designs, the resisting force of a continuum structure is an important 


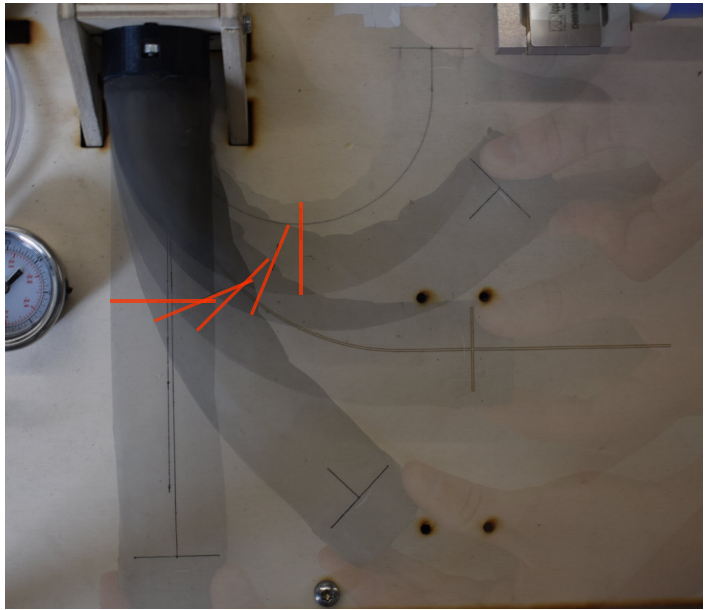

Fig. 5. Deformation of central diameter under bending, showing measured central diameter (hybrid - shared volume).

measurement to obtain, and combined with further measurements can be useful for comparison despite changes in size. To measure the resisting force, the structure is mounted horizontally at one end with stiffening activated, and the resistant force is measured by deforming the free end of the structure $10 \mathrm{~mm}$ perpendicular to the fixed end, thus applying a shear force, using a sensor attached to a linear actuator, which is then retracted to its initial position of $0 \mathrm{~mm}$ deformation. In our case a DBBSM 5kg S-Beam load cell and Actuonix L12-100-10012I linear actuator were used. This was performed with the link at $0^{\circ}, 90^{\circ}$, and $180^{\circ}$ bending, as shown in Fig. 3, reporting how the resisting force changes with bending angle. At $0^{\circ}$ the resisting force was also calculated with the link flexible, to demonstrate the force required to modify the topology of the structure. Each of these four experiments was repeated 10 times before being filtered (low pass) and averaged.

\section{B. Test 2: Positional Accuracy}

When undergoing a change from flexible to stiff, it is common for the structures to observe a positional change due to rearrangement of the internal structure. For specific use cases, e.g. as a manipulator, the ability to hold a set position is critical when changing stiffness. To measure the positional change, motion tracking markers were attached to the furthest end of the structure, and the motion of the structure as it underwent stiffening was measured using three motion tracking cameras (OptiTrack Flex 3), as shown in Fig. 4. In the setup of the tracking system, the calibration result report detailed the mean $3 \mathrm{D}$ error for overall projection as $0.111 \mathrm{~mm}$, with the overall wand error as $0.104 \mathrm{~mm}$. The structures were placed in their default linear form with no actively held positioning, based on the assumption that a maximum positional change was experienced when movement was not restricted. The motion in the $x$ and $y$ axes was recorded, with motion in the $z$ axis restricted by ensuring the structure was laid flat on a surface. The unmarked end of the structure was again mounted to prevent movement. The motion of each structure was repeated 5 times, and an average was calculated.

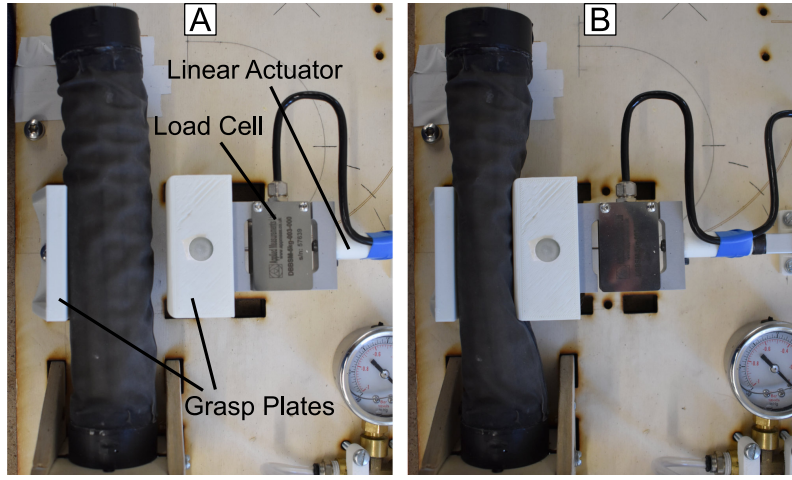

Fig. 6. Deformation of central diameter under external forces: (A) Before; (B) After (layer jamming).

\section{Test 3: Bending Diameter}

When undergoing bending, the ideal continuum manipulator maintains a constant diameter along its structure, with a neutral-line following down the central axis of the structure, as this allows for simplified theoretical analysis, positional control, and buckling avoidance, all which are critical in the use of continuum manipulators. This is a significant consideration for operation in confined areas, where large structural bending is expected, yet structural strength must be maintained. To measure the change in central diameter (the diameter at the mid-position along the structure), the continuum structure was mounted at one end, and bent manually from $0^{\circ}$ to $180^{\circ}$ in $45^{\circ}$ increments. At each angle the deformation was captured using a DSLR camera (Nikon D3400), and using image processing the change in diameter was measured based on the known, undeformed diameters. The bending angles, as well as the processed measurements of the central diameter, can be seen in Fig. 5. Each position was repeated 3 times to allow for an average to be taken.

\section{Test 4: Deformation under External Forces}

When manually deformed, that is, when manually handled by a user grasping the continuum structure, ideally no change in diameter along the structure is observed when an external force is applied, as a change in diameter indicates a loss in strength due to buckling [18]. However, for continuum structures composed of soft or delicate components, typically they exhibit a change in diameter when a force is imposed. To measure this change, with a focus on manual reshaping, each structure was mounted parallel to a fixed rigid plate of width $75 \mathrm{~mm}$, based off the lower boundary of average palm width [20]. A second equal sized plate, connected to a load cell, was pressed into the structure compressing the structure between the two plates, as shown in Fig. 6. The structure was compressed until both $10 \mathrm{~N}$ (gentle grasp) and 20N (medium grasp) of force were experienced [21], simulating a user imposing a grasp force on the structure. The deflection of the moving plate was tracked using motion tracking cameras (same calibration results are reported in section II B), resulting in the structural change in diameter for each grasp force. Compression of both grasps for each structure were repeated 5 times, to allow for an average. 


\section{E. Test 5: Cylindrical density and Activation Time}

The final experiment is composed of two smaller tests, as the results of the experiments are reported together in tabular form with other properties of the designs. The cylindrical density is a simplification of the density of each structure to allow for structures composed of multiple materials of varying densities. It also allows comparison across continuum structure sizes, without presenting issues with scaling. It is represented by the volume of each structure being assumed to be a cylinder with the outer dimensions of the structure, as the outer form of continuum structure is consistent across sizes. The cylindrical density is thus calculated using the weight of each structure divided by this volume. The second test determines the activation and deactivation time for transitioning between flexible and rigid modes. Optimal values for activation and deactivation time are as low as possible, as this ensures the position is activated in the desired position before moving, and further reduces the waiting time of a user. This was calculated using a pressure gauge to establish the pressure inside the stiffening volumes, and when activated the time taken to reach the desired held pressure of $-60 \mathrm{kPa}$ was recorded using an image capturing device to determine when the desired pressure was reached. For deactivation, the time taken for the internal pressure to return to $0 \mathrm{kPa}$ was recorded. The activation and deactivation were each repeated 5 times for an average to be taken.

\section{EXPERIMENTAL RESULTS}

Each of the 5 experiments were carried out on the 6 continuum designs, and the results were collected using MATLAB software. The results for each experiment are detailed in their respective subsections:

\section{A. Force Resistance}

The measured resisting force for a given deflection of the end of the continuum structure can be seen in Fig. 7. Each of the 4 sub-figures show variations in stiffness (fleixble/rigid) and bending angle $\left(0^{\circ}, 90^{\circ}, 180^{\circ}\right)$. Error bars are also shown for each plot indicating \pm 1 standard deviation every $1 \mathrm{~mm}$ deflected. To ensure distinguishability between structures on a plot, the Y-axis limits vary between sub-figures. The results were aligned for each plot by measuring the initial offset of the load cell, and forward [Fig. 7(left)] and reverse [Fig. 7(right)] plots were aligned together using the software Affinity Photo. As the load cell was retracted, shown in the reverse plots, the force either decreased to $0 \mathrm{~N}$, indicating a positional hysteresis of the structure holding its deflection, or until the initial $0 \mathrm{~mm}$ deflection point was reached.

From the plots we can observe that, when flexible, the hybrid - shared volume design required the largest force to deflect, at $0.8 \mathrm{~N}$, whereas layer jamming and deformable granules exhibited the lowest force at $0.2 \mathrm{~N}$. When retracted, all of the designs showed a very similar hysteresis holding a deflection of $\sim 9 \mathrm{~mm}$. When rigid, the majority of the structures performed consistently, with others showing variation dependent on the bending angle: The highest resisting force for each of the structures was shown at $180^{\circ}$ bending, except
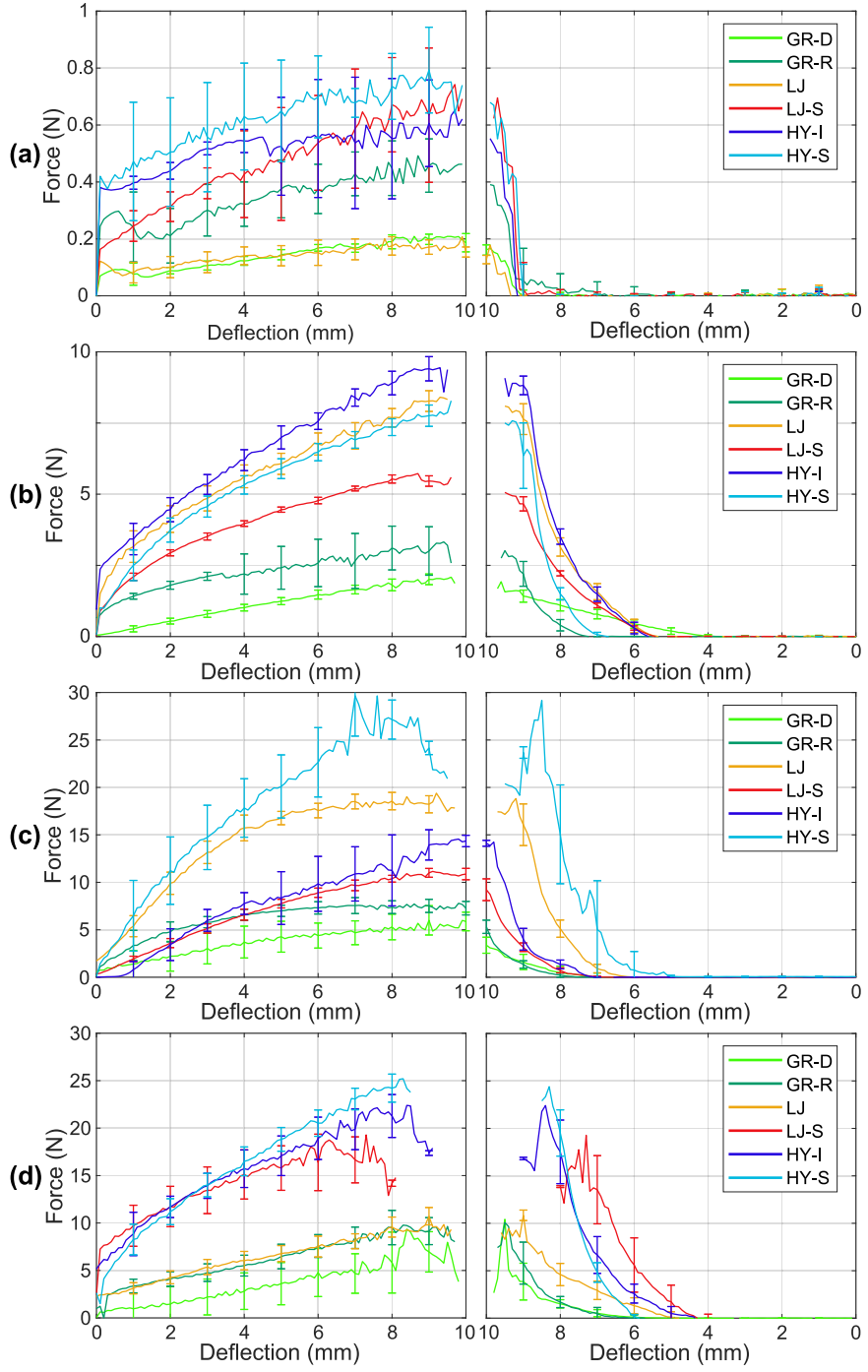

Fig. 7. Resisting force vs. displacement plots for stiffness modes and bending positions. (a) flexible $0^{\circ}$, (b) rigid $0^{\circ}$, (c) rigid $90^{\circ}$, and (d) rigid $180^{\circ}$.

for layer jamming and hybrid - shared volume who showed their highest resisting force at $90^{\circ}$ at $19.4 \mathrm{~N}$ and $29.7 \mathrm{~N}$, respectively. In all cases the granular designs performed worse than layer jamming-based designs, and further barring $0^{\circ}$ rigid deformable granules demonstrated a higher hysteresis in all cases. For the rigid cases, the highest resisting force was demonstrated by hybrid - individual volumes at $0^{\circ}(9.4 \mathrm{~N})$, hybrid - shared volume at $90^{\circ}(29.7 \mathrm{~N})$, and hybrid - shared volume at $180^{\circ}(25.2 \mathrm{~N})$.

\section{B. Positional Accuracy}

From the averages collected the positional changes in $x$ and $y$ axes were plotted, as well as lines indicating the change from 0, shown in Fig. 8. The activation of deformable granules resulted in the highest change in the $y$ axis, showing a large reduction in length of $\sim 11 \mathrm{~mm}$. Rigid granules demonstrated the highest positional accuracy, showing the smallest change with $[0.05 \mathrm{~mm}, 0.03 \mathrm{~mm}]$ in the $[x, y]$ axis, followed by layer jamming with internal support at $[-0.22 \mathrm{~mm},-0.10 \mathrm{~mm}]$. In contrast, both hybrid designs showed the highest change in 


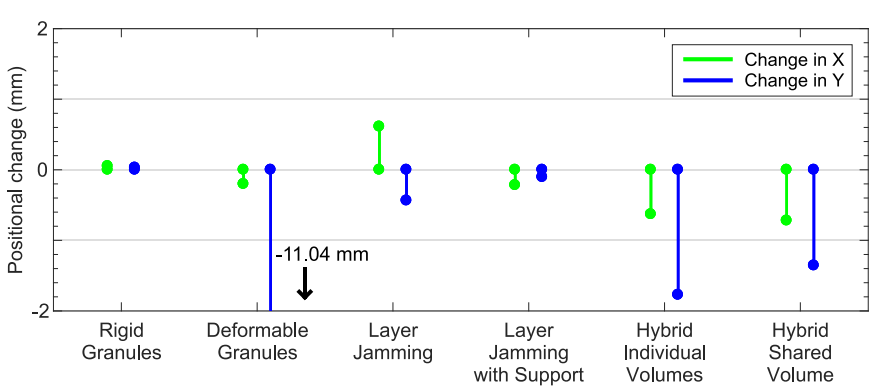

Fig. 8. Positional accuracy of continuum structure after stiffening, represented as distance moved in the $x$ axis (green) and $y$ axis (blue).

the $x$ axis at $-0.63 \mathrm{~mm}$ and $-0.72 \mathrm{~mm}$ for hybrid - individual volumes and hybrid - shared volume, respectively.

\section{Central Diameter Variation}

The central diameter variation at bending angles from $0^{\circ}$ to $180^{\circ}$ can be seen in Fig. 9. The digitally measured diameters from $45^{\circ}$ to $180^{\circ}$ were converted to a ratio of the initial $\left(0^{\circ}\right)$ diameter, which were then plotted to show the change in diameter as each structure was bent. Layer jamming demonstrated the highest change in bending diameter, with $79.8 \%$ of the initial diameter at $180^{\circ}$. On average granular jamming-based structures performed better than alternatives. However, layer jamming with internal support structure performed the best, showing the smallest ratio change of 0.964 at $180^{\circ}$.

\section{Deformation under External Forces}

The central diameter deformations of each structure under external forces at $10 \mathrm{~N}$ and $20 \mathrm{~N}$ (low and medium force grasps, respectively), along with marked anomalies (i.e., data outside 99.3\% coverage of a normal distribution), are displayed in Fig. 10 as box plots. The median is marked by the black line within the upper and lower quartiles, indicated by a coloured box (red for $10 \mathrm{~N}$, blue for $20 \mathrm{~N}$ ), with the upper and lower limits of valid data given by black whiskers. The lowest change in diameter was demonstrated by layer jamming with support, at $1.9 \mathrm{~mm}$ and $2.4 \mathrm{~mm}$ for $10 \mathrm{~N}$ and $20 \mathrm{~N}$, respectively, whereas the highest change in diameter was experienced by layer jamming, showing a maximum deformation of $23.0 \mathrm{~mm}$ at $20 \mathrm{~N}$. All continuum structures presented a higher deformation with the higher force grasp, with rigid granules and layer jamming showing the largest difference between the $10 \mathrm{~N}$ and $20 \mathrm{~N}$ grasp forces.

\section{E. Cylindrical Density and Stiffening Duration}

The calculated cylindrical density, along with the average activation and deactivation time, can be seen in Table I alongside other physical and dimensional properties for the continuum structures. Using the measured diameter, weight, and length of each structure the cylindrical densities were computed, with deformable granules showing the lowest density at $4.48 \times 10^{-3} \mathrm{~g} \mathrm{~mm}^{-3}$, and rigid granules showing the highest density at $39.42 \times 10^{-3} \mathrm{~g} \mathrm{~mm}^{-3}$. Both hybrid designs demonstrated a high density, whereas the layer jamming-based designs gave similar densities of $\sim 10 \times 10^{-3} \mathrm{~g} \mathrm{~mm}^{-3}$, with pure layer jamming showing a slightly lower density. For the activation and deactivation time, layer jamming took the shortest period to both activate and deactivate at $1.94 \mathrm{~s}$ and $1.18 \mathrm{~s}$, respectively. The highest activation and deactivation time was shown by deformable granules at $3.69 \mathrm{~s}$ and $2.59 \mathrm{~s}$. Both layer jamming designs averaged similar activation and deactivation times, with hybrid designs on average showing a slightly slower time to activate and deactivate.

\section{DISCUSSION}

The comprehensive experiments performed on the developed prototypes produce many interesting results for continuum structures. From the resisting force, we observe that a low force of $<1 \mathrm{~N}$ is required to deform all of the structures, with layer jamming with support, rigid granules, and hybrid designs requiring $2.4-3.8 \times$ the force needed to deform pure layer jamming and deformable granules. Despite the differences exhibited in resisting force, all prototypes showed a hysteresis of $\sim 9 \mathrm{~mm}$ on force retraction, most likely attributed to the elastic material properties of the membrane used. For the resisting forces at $0^{\circ}, 90^{\circ}$, and $180^{\circ}$, all designs except for layer jamming demonstrated an increase in resisting force with bending angle, which can be attributed to the buckling of the layer jamming at high angles [18].

Granular designs presented the weakest forces, giving a maximum resisting force from rigid granules at $180^{\circ}$ of $9.8 \mathrm{~N}$. Of the granular designs, rigid granules consistently performed better, due to the inelastic nature of the granules compared to deformable granules. The inclusion of support in layer jamming maintained the resisting force at $180^{\circ}$, and hybrid designs combining rigid granules with layer jamming also experienced a similar result. However, other than at $0^{\circ}$, the hybrid-shared volume performed better than hybrid-individual volumes, demonstrating a significantly higher resisting force at $90^{\circ}$ of $29.7 \mathrm{~N}$ compared to $14.6 \mathrm{~N}$ of the hybrid-individual volumes, an increase of $103.7 \%$. Hybrid-shared volume also demonstrated the highest resisting force in all cases except for $0^{\circ}$ rigid. In all of the resisting force experiments a hysteresis was shown, with the lowest hysteresis demonstrated by deformable granules at $0^{\circ}$, attributing to the elasticity of the structure.

Considering the positional accuracy, where a more accurate position after stiffening results in better control of a manipulator, we observe that deformable granules are not suitable for such a task due to their high change in length (decrease of $11 \mathrm{~mm}$ ) when transforming from flexible to rigid. All of the designs showed little change in the $x$ axis, with all positional changes in $x$ less than $\pm 1 \mathrm{~mm}$. Rigid granules and layer jamming with support demonstrated very little positional change due to the lack of ability to compress, thanks to the rigid granules and support structure contained within. Both hybrid designs exhibited a larger change in the $y$ axis at $\sim-2 \mathrm{~mm}$, likely attributed to air gaps contained between the granules and the layer jamming compressing when activated. Ideally this would be solved by the improved filling of the rigid granules in the hybrid designs, however it is expected 


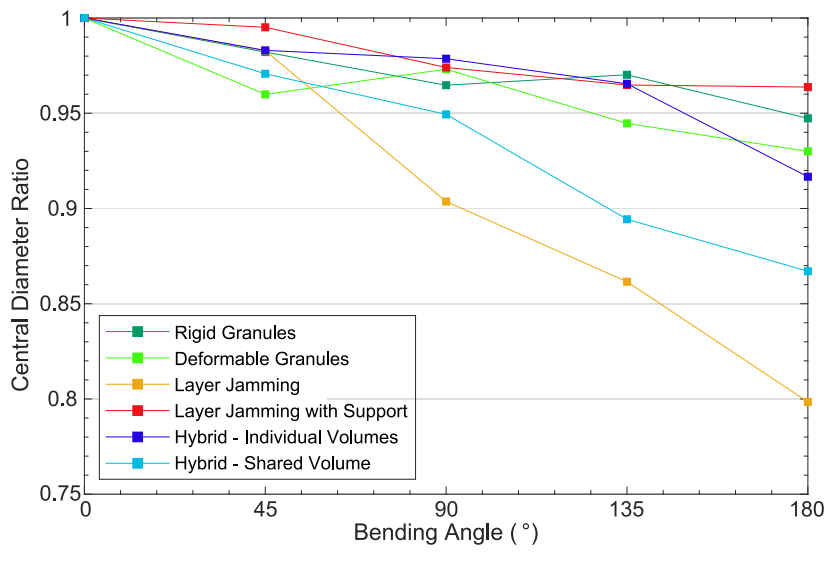

Fig. 9. Variation of central diameter with bending angle.

this would reduce the bending ability but increase the resistant force.

The variation of central diameter under bending gives and impression of the bending ability of the link, with more consistent designs indicating a higher bending ability, as well as higher simplicity in simulation of the continuum structure topology. Layer jamming, lacking an internal structure compared to the other designs, showed the lowest central diameter ratio, decreasing to $79.84 \%$ as the structure buckled at high bending angles. Layer jamming with support, rigid granules, deformable granules, and hybrid-individual volumes all demonstrated similar reductions in central diameter to $92-$ $96 \%$, with layer jamming with support maintaining the highest central diameter at $180^{\circ}$ at $96.37 \%$. Hybrid-shared volume however, demonstrated a large decrease to $86.71 \%$. We believe this can be explained by the movement of the rigid granules in the structure, leading to the slight buckling of the layer jamming aspect into the free space. This corresponds with the findings from the positional accuracy of the inclusion of air pockets, and suggests the included membrane in the hybridindividual volumes designs helps to resist the movement of the granules preventing buckling of the layer jamming.

Considering the deformation of the diameter under external forces, that is, gentle grasping at $10 \mathrm{~N}$ and medium grasping at $20 \mathrm{~N}$, we observe similar results to the central diameter variation, as well as different results further explaining the performance of the structures. With layer jamming we observe the largest change in diameter for both the $10 \mathrm{~N}$ and $20 \mathrm{~N}$ grasp, as is expected from the large change in bending diameter ratio and ability to buckle, showing a change of $15.3 \mathrm{~mm}$ and $23.0 \mathrm{~mm}$ for each grasp, respectively. Also expected, layer jamming with support demonstrates the lowest change in diameter due to the internal structure preventing deformation, giving a very low change of $1.9 \mathrm{~mm}$ and $2.4 \mathrm{~mm}$ for $10 \mathrm{~N}$ and $20 \mathrm{~N}$, respectively. In comparison, rigid granules and deformable granules demonstrated a high change in diameter under grasping due to the grasp force overcoming the limit needed to move the granules, whereas under bending the force produced by the membrane was not high enough to overcome this limit, and thus the diameter ratio was maintained. A similar deformation of $\sim 11.5 \mathrm{~mm}$ was observed for both granular designs at

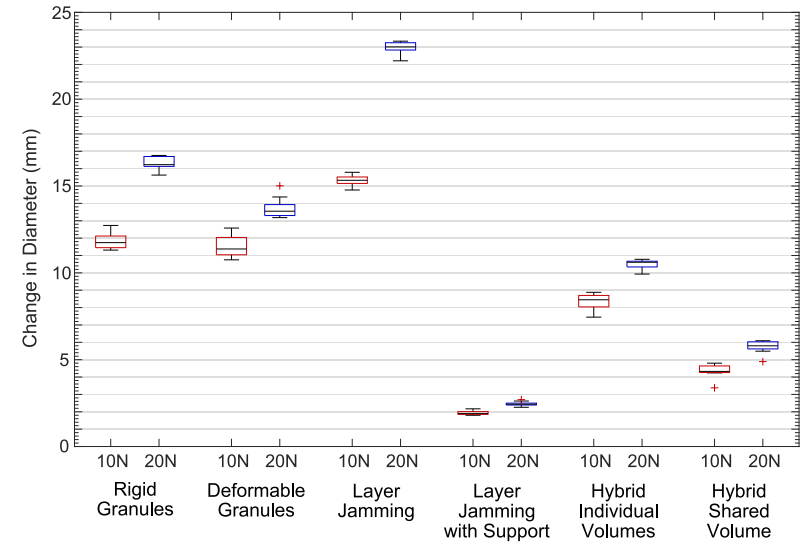

Fig. 10. Deformation of diameter under a low $(10 \mathrm{~N})$ and medium $(20 \mathrm{~N})$ force grasp.

$10 \mathrm{~N}$, however a higher deformation of $16.2 \mathrm{~mm}$ compared to $13.6 \mathrm{~mm}$ was observed by the rigid granules at $20 \mathrm{~N}$, explained by deformable granules approaching their compression limit, compared to the rigid granules instead moving out of the way of the grasp. Both hybrid designs performed better than granular, with the shared volume presenting a lower change in diameter, indicating a lower number of air gaps, and therefore a better granule filling, within the structure.

Finally, we consider the dimensional and physical properties of the continuum structures. Of the cylindrical density, the deformable granules and layer jamming exhibit the lowest values at $4.48 \times 10^{-3} \mathrm{~g} \mathrm{~mm}^{-3}$ and $8.70 \times 10^{-3} \mathrm{~g} \mathrm{~mm}^{-3}$, respectively. The low density of the deformable granules, as well as the hollow nature of the layer jamming, attribute to the low density. The highest cylindrical density was achieved by the rigid granules as expected due to their high weight at $39.42 \times 10^{-3} \mathrm{~g} \mathrm{~mm}^{-3}$. Both hybrid designs utilising rigid granules also demonstrated a high cylindrical density. The inclusion of an internal support structure in layer jamming increased the cylindrical density by $31 \%$. Depending on the application and purpose, it may be useful to normalise the maximum resistant force using the cylindrical density to facilitate comparison between designs. The activation and deactivation time tests resulted in layer jamming and layer jamming with support achieving the lowest activation and deactivation time, due to the reduced volume being evacuated as well as the lack of gaps partially trapping air. This explains the increased times observed with both granular designs showing high activation times, attributed to the partially trapped air compressing the volume and having difficulty evacuating. This effect can be seen in reverse with the deformable granules, where once compressed required the largest deactivation time for the air to fill the reduced spaces. The other structural designs exhibited similar activation and deactivation times, at $\sim 2.5 \mathrm{~s}$ for activation and $\sim 1.7 \mathrm{~s}$ for deactivation.

The variety of metrics available in these tests allows for the selection of an optimal technology based on the specific desired use case. For instance, in a manually adjusted continuum structure-relevant in industrial settings where it can be used as a component of a collaborative manipulator, where high 
TABLE I

DiMENSIONAL AND PHYSICAL PROPERTIES FOR THE DEVELOPED CONTINUUM STRUCTURES

\begin{tabular}{lcccccc}
\hline Type & Stiffening Method & $\begin{array}{c}\text { Diameter } \\
(\mathrm{mm})\end{array}$ & $\begin{array}{c}\text { Weight } \\
(\mathrm{g})\end{array}$ & $\begin{array}{c}\text { Cylindrical Density } \\
\left(\mathrm{g} \mathrm{mm}^{-3}\right)\end{array}$ & $\begin{array}{c}\text { Activation } \\
\text { Time }(\mathrm{s})\end{array}$ & $\begin{array}{c}\text { Deactivation } \\
\text { Time }(\mathrm{s})\end{array}$ \\
\hline Rigid Granules & Granular & 48 & 438 & $39.42 \times 10^{-3}$ & 2.58 & 1.63 \\
Deformable Granules & Granular & 48 & 50 & $4.48 \times 10^{-3}$ & 3.69 & 2.60 \\
Layer Jamming & Layers & 48 & 96 & $8.70 \times 10^{-3}$ & 1.94 & 1.18 \\
Layer Jamming with Support & Layers & 41 & 116 & $11.37 \times 10^{-3}$ & 1.97 & 1.19 \\
Hybrid - Individual Volumes & Granular \& Layers & 48 & 300 & $32.34 \times 10^{-3}$ & 2.89 & 1.83 \\
Hybrid - Shared Volume & Granular \& Layers & 48 & 376 & $26.82 \times 10^{-3}$ & 2.09 & 1.65 \\
\hline
\end{tabular}

strength with little structural variation is desired, we observe both hybrid designs as performing particularly well with high resisting forces, however with higher structural deformations under bending and grasping and less positional accuracy than an alternative design. Layer jamming with support would be the ideal selection here, from the minimal structural changes, and consistent resisting force and flexible-to-stiff transition.

\section{CONCLUSION}

In this paper we presented five different metrics for establishing the performance of variable stiffness continuum structures. The methods described vary in importance according to the requirement of the targeted application, such as the need of high force resistance or ability for manual handling. We evaluated the performance of six prototypes using both typical (granular and layer jamming) and novel designs (hybrid and supported). The prototype using hybrid jamming in a shared volume performed best for resistant force focused tasks, with a maximum resisting force of $29.7 \mathrm{~N}$ demonstrated, whereas for a task focused on human interaction layer jamming with internal support performed best showing the smallest reduction in diameter under an external force $(2.4 \mathrm{~mm}$ at $20 \mathrm{~N})$, and the smallest change in diameter when undergoing bending (reduction of $3.63 \%$ ). All testing methodologies presented resulted in a distribution of performances, indicating the selection of metrics cover a variety of advantages and use cases for continuum structures. The methodology presented can be applied across other stiffening technologies, such as phase transition or rheological fluids, providing a comparison across various sizes and methods of variable stiffness. Future work might centre on further analysis of varying sizes of continuum structures or the comparison of other variable stiffness solutions.

\section{REFERENCES}

[1] A. Jiang, G. Xynogalas, P. Dasgupta, K. Althoefer, and T. Nanayakkara, "Design of a Variable Stiffness Flexible Manipulator with Composite Granular Jamming and Membrane Coupling", IEEE/RSJ IROS, pp. 2922-2927, 2012.

[2] J. L. C. Santiago, I. D. Walker, and I. S. Godage, "Continuum Robots for Space Applications based on Layer-Jamming Scales with Stiffening Capability", Proceedings of the 2015 IEEE Aerospace Conference, pp. 1-13, 2015.

[3] Y-J. Kim, S. Cheng, S. Kim, and K. Iagnemma, "A Novel Layer Jamming Mechanism with Tunable Stiffness Capability for Minimally Invasive Surgery", IEEE Transactions on Robotics, vol. 29, no. 4, pp. 1031-1042, 2013

[4] E. Thompson-Bean, O. Steiner, and A. McDaid, "A Soft Robotic Exoskeleton utilizing Granular Jamming", IEEE AIM, pp. 165-170, 2015.
[5] R. Kang, D. T. Branson, T. Zheng, E. Guglielmino, and D. G. Caldwell, "Design, Modeling and Control of a Pneumatically Actuated Manipulator inspired by Biological Continuum Structures", Bioinspiration \& Biomimetics, vol. 8, no. 3, pp. 036008, 2013.

[6] M. D. Grissom, V. Chitrakaran, D. Dienno, M. Csencits, M. Pritts, B. Jones, W. McMahan, D. Dawson, C. Rahn, and I. Walker, "Design and Experimental Testing of the Octarm Soft Robot Manipulator", SPIE: Unmanned Systems Technology VIII, vol. 6230, pp. 62301F, 2006.

[7] W. McMahan, B. A. Jones, and I. D. Walker, "Design and Implementation of a Multi-section Continuum Robot: Air-Octor", IEEE/RSJ IROS, pp. 2578-2585, 2005.

[8] M. A. Robertson, H. Sadeghi, J. M. Florez, and J. Paik, "Soft Pneumatic Actuator Fascicles for High Force and Reliability", Soft Robotics, vol. 4, no. 1, pp. 23-32, 2017.

[9] J. Burgner-Kahrs, D. C. Rucker, and H. Choset, "Continuum Robots for Medical Applications: A Survey", IEEE Transactions on Robotics, vol. 31, no. 6, pp. 1261-1280, 2015.

[10] J. L. C. Santiago, I. S. Godage, P. Gonthina, and I. D. Walker, "Soft Robots and Kangaroo Tails: Modulating Compliance in Continuum Structures through Mechanical Layer Jamming”, Soft Robotics, vol. 3, no. 2, pp. 54-63, 2016.

[11] L. Blanc, A. Delchambre, and P. Lambert, "Flexible Medical Devices: Review of Controllable Stiffness Solutions", MDPI Actuators, vol. 6, no. 3, pp. 23, 2017.

[12] Y. Kitagawa and T. Mitsuda, "Variable stiffness element by granular jamming (Stiffness enhancement by a wrinkle-free outer membrane)", Transactions of the JSME, vol. 83, no. 851, pp. 17-00107, 2017.

[13] M. Manti, V. Cacucciolo, and M. Cianchetti, "Stiffening in Soft Robotics: a Review of the State of the Art", IEEE Robotics \& Automation Magazine, vol. 23, no. 3, pp. 93-106, 2016.

[14] W. McMahan, B. Jones, I. Walker, V. Chitrakaran, A. Seshadri, and D. Dawson, "Robotic Manipulators inspired by Cephalopod Limbs", Proceedings of the 2004 CDEN Design Conference, pp. 1-10, 2004.

[15] A. Shiva, A. Stilli, Y. Noh, A. Faragasso, I. D. Falco, G. Gerboni, M. Cianchetti, A. Menciassi, K. Althoefer, and H. A. Wurdemann, "Tendonbased Stiffening for a Pneumatically Actuated Soft Manipulator", IEEE $R A L$, vol. 1, no. 2, pp. 632-637, 2016.

[16] V. Wall, R. Deimel, and O. Brock, "Selective Stiffening of Soft Actuators based on Jamming", IEEE ICRA, pp. 252-257, 2015.

[17] M. Langer, E. Amanov, and J. Burgner-Kahrs, "Stiffening sheaths for continuum robots", Soft Robotics, 2018.

[18] A. B. Clark and N. Rojas, "Stiffness-Tuneable Limb Segment with Flexible Spine for Malleable Robots", IEEE ICRA, 2019, Accepted.

[19] J. Ruiz-Ruiz, J. L. M. Mesa, A. Gutiérrez, and M. J. Castillo, "Hand Size Influences Optimal Grip Span in Women but not in Men", The Journal of Hand Surgery, vol. 27, no. 5, pp. 897-901, 2002.

[20] C. W. Nicolay and A. L. Walker, "Grip Strength and Endurance: Influences of Anthropometric Variation, Hand Dominance, and Gender", International Journal of Industrial Ergonomics, vol. 35, no. 7, pp. 605$618,2005$.

[21] R. Calandra, A. Owens, D. Jayaraman, J. Lin, W. Yuan, J. Malik, E. H. Adelson, and S. Levine, "More Than a Feeling: Learning to Grasp and Regrasp using Vision and Touch", IEEE RAL, Preprint, 2018. 